\title{
Prophylactic Central Neck Dissection Increases the Cost of Thyroid Cancer Care
}

\author{
Dawn M. Elfenbein, MD, MPH and Rebecca S. Sippel, MD \\ Section of Endocrine Surgery, Department of Surgery, University of Wisconsin, Madison, WI
}

Over the past several decades, health care spending has risen faster than any other segment of the United States economy and continues to rise. ${ }^{1}$ Media attention has been focused on health care costs since the introduction of the Patient Protection and Affordable Care Act of 2010, which aims to reduce costs of health care while improving outcomes. Physicians are in the best position to determine which treatments are effective and which patients benefit most, and they are more cognizant than ever of the mandate to spend our limited health care dollars wisely. Cost minimization should be the goal of every physician, as long as quality of care does not diminish.

The incidence of papillary thyroid cancer (PTC) is increasing in the United States, but thankfully survival is excellent compared with many other cancers. Patients diagnosed when the disease is limited to the thyroid gland are nearly always cured with thyroidectomy. Unfortunately, regional lymph node metastases from PTC are very common, with the majority of patients having at least micrometastatic disease in the central neck at the time of diagnosis. Nodal involvement increases the risk of recurrence, which plays an important role in the long-term management of this disease. While there is no debate about removing clinically apparent lymph node disease at the initial surgery, there is significant debate regarding the utility of performing a prophylactic central lymph node dissection (pCND) as part of the initial thyroidectomy

This is an editorial to the article available at doi: $10.1245 / \mathrm{s} 10434-$ 013-3234-9.

\section{(C) Society of Surgical Oncology 2013}

First Received: 10 September 2013; Published Online: 8 October 2013

R. S. Sippel, MD

e-mail: sippel@surgery.wisc.edu when there is no evidence of lymph node involvement. There has not been a definitive randomized controlled trial to date, and there are strong advocates on both sides of the argument. ${ }^{2}$ Proponents claim pCND saves future morbidity of repeat operations without adding much operative risk to the initial operation, while opponents feel it adds operative morbidity without altering long-term outcomes for the majority of patients. Most of the available literature has struggled to address either the risk or the benefit of pCND, and there has been no consensus. Lang et al., ${ }^{3}$ in this current issue, aim to answer the question from a costeffectiveness perspective, adding yet another dimension to this debate at a timely moment in national discourse.

They report, unsurprisingly, that the initial procedural costs are higher for pCND at the time of initial surgery for node-negative PTC versus performing a simple thyroidectomy alone. The key question is whether that initial investment (and higher costs of more initial complications) saves health care spending in the long term by reducing the rates of reoperations and future complications. Using data from their recent well-done meta-analysis of 14 studies with 3,331 patients, the authors used odds ratios for complications of both initial surgeries, recurrence rates, and the likelihood of radioactive iodine (RAI) treatment to create a mathematical model. ${ }^{4}$ This model projected out costs for a hypothetical cohort of 100,000 women with a $1.5-\mathrm{cm}$ papillary thyroid cancer. Using it, they found that pCND is not cost saving and calculated that, over 20 years, the accumulated direct costs of a thyroidectomy with pCND is $\$ 3000$ more than initially performing a thyroidectomy alone and dealing with recurrences as they arise.

In this hypothetical cohort of patients, the higher costs of pCND are mostly attributable to (1) longer operative time and greater complexity of the operation, (2) more initial complications, and (3) higher percentage of postoperative RAI in patients with pCND. Reoperations actually have similar costs in this model, and while fewer 
reoperations are required for recurrence in the $\mathrm{pCND}$ group, that does not make up for the higher initial costs. Being a cost-effectiveness study, the current analysis considers only direct costs, but the reader should keep in mind that there are other costs including loss of wages, as well as the impact of complications or future recurrences on patient quality of life that are not a part of this equation. It seems intuitive that the first 2 reasons (more surgery and greater complications) lead to increased cost; however, the careful reader should keep in mind that this model is based on published complication rates. Not only is there massive variation in published rates of both complications and recurrences, but there is always a publication bias toward good outcomes, so the true rates of complication are not well known. Additionally, most published studies are performed at high-volume centers with experienced endocrine surgeons, yet the majority of thyroid surgery is performed by general surgeons and otolaryngologists in the community. The third point, regarding the use of RAI, is probably the most important issue to consider as it has a huge impact on the costs of care, and we think that warrants further discussion.

Consider a hypothetical world where RAI therapy does not exist: Surgical removal of lymph nodes that harbor micrometastatic disease would lead to fewer recurrences than leaving lymph node microscopic disease in situ. Yet, in current practice, it is precisely these patients who undergo more complete surgery and should have lower recurrence rates without RAI who are getting more RAI therapy (77 vs $53 \%$ for the current Lang and Wong analysis). This should seem counterintuitive, yet it is shown time and time again in practice. ${ }^{5}$ The reason for this is the phenomena of pathological upstaging: If nodes are clinically negative, those left in situ remain unexamined by a pathologist's microscope and the patient's stage remains N0, while nodes that are removed and examined can upstage the patient's nodal status. Instead of interpreting the pathology report that contains micrometastatic disease in nodes as a positive prognostic factor (as in, all of the PTC has been removed), it is interpreted as a higher-risk feature (as in, the PTC has spread to the lymph nodes), even though those patients without pCND have the same rates of uninspected micrometastatic disease. According to the official ATA recommendations for RAI therapy, conflicting data exist, and the recommendation is for "selective use" in patients with N1 disease-that is, the official guidelines recommend neither for nor against RAI, but encourage practitioners to consider RAI in higher-risk patients, of which nodal disease is one risk factor. ${ }^{6}$ In practice, RAI renders thyroglobulin (Tg) levels undetectable, and a bump in that level alerts the clinician to the possibility of recurrence and that further workup is indicated. Omitting RAI postoperatively leads to a low but detectable $\mathrm{Tg}$ level, and small fluctuations are more difficult to interpret. Until clinicians are more comfortable following patients with low but detectable $\mathrm{Tg}$ levels, overuse of RAI will continue to add significant expense to the treatment of PTC. If the use of pCND could actually be used to decrease our use of RAI, then the cost effectiveness of this intervention could easily swing in favor of pCND.

There is still no consensus on whether pCND at the time of initial thyroidectomy for clinically node-negative PTC leads to better long-term outcomes with acceptable operative risk. In areas such as this, where there is no overwhelming evidence for benefit, the costs of the intervention must be carefully considered, especially in the current era of maximizing efficiency in health care spending. We can no longer afford to spend money that is not going to have a real clinical benefit, and the current study by Lang and Wong is an attempt to clarify the costs of this practice using current published data. Unfortunately, current published data contains large amounts of variation and bias, as well as practice patterns of RAI administration that probably do not reflect the most cost-effective use management of thyroid cancer. A well-designed, randomized controlled trial could help answer this debate, but only after many years of follow-up. Until then, cost is an important factor to consider in future studies on this complicated issue.

\section{REFERENCES}

1. Emanuel E, Tanden N, Altman S, Armstrong S, Berwick D, de Brantes F, et al. A systemic approach to containing health care spending. New Engl J Med. 2012;367:949-54.

2. Carling T, Carty SE, Ciarleglio MM, Cooper DS, Doherty GM, Kim LT, et al. American Thyroid Association design and feasibility of a prospective randomized controlled trial of prophylactic central lymph node dissection for papillary thyroid carcinoma. Thyroid. 2012;22:237-44.

3. Lang $\mathrm{BH}$, Wong $\mathrm{CK}$. A cost-minimization analysis comparing total thyroidectomy alone and total thyroidectomy with prophylactic central neck dissection in clinically nodal-negative papillary thyroid carcinoma. Ann Surg Oncol. 2013. doi:10.1245/s10434013-3234-9.

4. Lang BH, Ng SH, Lau LL, Cowling BJ, Wong KP, Wan KY. A systematic review and meta-analysis of prophylactic central neck dissection on short-term locoregional recurrence in papillary thyroid carcinoma after total thyroidectomy. Thyroid. 2013;23: 1087-98.

5. Wang TS, Evans DB, Fareau GG, Carroll T, Yen TW. Effect of prophylactic central compartment neck dissection on serum thyroglobulin and recommendations for adjuvant radioactive iodine in patients with differentiated thyroid cancer. Ann Surg Oncol. 2012;19:4217-22.

6. Cooper DS, Doherty GM, Haugen BR, Kloos RT, Lee SL, Mandel SJ, et al. Revised American Thyroid Association management guidelines for patients with thyroid nodules and differentiated thyroid cancer. 2009;19:1167-214. 